\title{
Metallic phase transition metal dichalcogenide quantum dots showing different optical charge excitation and decay pathways
}

\author{
Bo-Hyun Kim (1), Min-Ho Jang ${ }^{2}$, Hyewon Yoon ${ }^{3}$, Hyun Jun Kim', Yong-Hoon Cho², Seokwoo Jeon $\mathbb{1}^{3}$ and \\ Sung-Ho Song $\mathbb{1 D}^{1}$
}

\begin{abstract}
The charge excitation and decay pathways of two-dimensional heteroatomic quantum dots (QDs) are affected by the quantum confinement effect, bandgap structure and strong exciton binding energy. Recently, semiconducting transition metal dichalcogenides (TMDs) have been intensively studied; however, the charge dynamics of metallic phase QDs (mQDs) of TMDs remain relatively unknown. Herein, we investigate the photophysical properties of TMD$m Q D$ s of two sizes, where the TMD-mQDs show different charge excitation and decay pathways that are mainly ascribed to the defect states and valence band splitting, resulting in a large Stokes shift and two excitation bands for maximum photoluminescence (PL). Interestingly, the dominant excitation band redshifts as the size increases, and the time-resolved PL peak redshifts at an excitation wavelength of $266 \mathrm{~nm}$ in the smaller QDs. Additionally, the lifetime is shortened in the larger QDs. From the structural and theoretical analysis, we discuss that the charge decay pathway in the smaller QDs is predominantly affected by edge oxidation, whereas the vacancies play an important role in the larger QDs.
\end{abstract}

\section{Introduction}

Following extensive studies on two-dimensional (2D) layered materials, transition metal dichalcogenide (TMD)based quantum dots (QDs) have attracted significant attention owing to their potential applications in optoelectronics, catalysis, biosensors, energy systems, etc ${ }^{1-4}$. The different structural phases, i.e., semiconducting $(2 \mathrm{H})$ and metallic $\left(1 \mathrm{~T} / 1 \mathrm{~T}^{\prime}\right)$ phases, of $2 \mathrm{D}$ TMDs with stoichiometric combinations $\left(\mathrm{MX}_{2}\right)$ of transition metals $(\mathrm{M}=\mathrm{Mo}, \mathrm{W}, \mathrm{Ni}$, etc. $)$ and chalcogens $(\mathrm{X}=\mathrm{S}, \mathrm{Se}, \mathrm{Te})$ exhibit various electronic and optical properties ${ }^{5-8}$. The electronic and optical properties of TMD-QDs are usually explained by the combined mechanism of the quantum

\footnotetext{
Correspondence: Seokwoo Jeon (jeon39@kaist.ac.kr) or

Sung-Ho Song (shsong805@kongju.ac.kr)

'Division of Advanced Materials Engineering, Kongju National University, Chungnam 31080, South Korea

²Department of Physics and KI for the NanoCentury, KAIST, Daejeon 34141, South Korea

Full list of author information is available at the end of the article
}

confinement effect, surface state, and doping effect ${ }^{9-11}$, which is also sensitive to environmental conditions, chalcogen defects, and heteroatomic crystal structures $^{4,12,13}$. As the QDs form a monolayer, the electronic band structure widens to $\sim 3-5 \mathrm{eV}$, with slight variations depending on the type of transition metal and chalcogen ${ }^{13,14}$. Furthermore, TMD-QDs exhibit a strong exciton binding energy and significantly enhanced spin-valley coupling, which induces broadening of excitonic absorption peaks and generates a higher quantum yield compared to the flake types ${ }^{2-4}$. $\mathrm{MoSe}_{2}$ - and $\mathrm{WS}_{2}-\mathrm{QDs}$ show an excitation wavelength-dependent photoluminescence (PL) shift, suggesting two different recombination pathways of excitons ${ }^{15,16}$. However, to date, the optical properties of metallic phase TMD-QDs (TMD-mQDs) compared to those of semiconducting TMD-QDs have been ambiguous and not consistently reported ${ }^{4,17-19}$.

The spectroscopic results for metallic TMDs show additional Raman modes below $200 \mathrm{~cm}^{-1}$, a monotonic

\section{(c) The Author(s) 2021}

(c) (i) Open Access This article is licensed under a Creative Commons Attribution 4.0 International License, which permits use, sharing, adaptation, distribution and reproduction cc) in any medium or format, as long as you give appropriate credit to the original author(s) and the source, provide a link to the Creative Commons license, and indicate if changes were made. The images or other third party material in this article are included in the article's Creative Commons license, unless indicated otherwise in a credit line to the material. If material is not included in the article's Creative Commons license and your intended use is not permitted by statutory regulation or exceeds the permitted use, you will need to obtain permission directly from the copyright holder. To view a copy of this license, visit http://creativecommons.org/licenses/by/4.0/. 


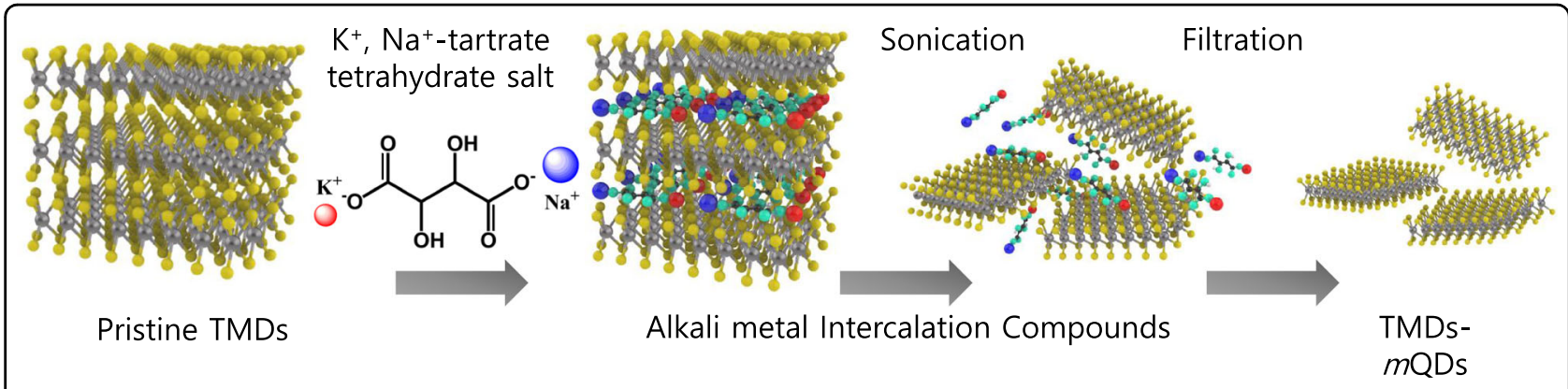

Fig. 1 Fabrication route for TMD-QDs. Schematic illustration of the synthetic process of TMDs-mQDs. The synthetic route is composed of three parts: forming compounds, making QDs, and filtering. Yellow and gray represent chalcogens and transition metals.

change in UV-visible absorption, and a shift in photoelectron peaks ${ }^{6,20,21}$. TMD- $m$ QDs that are electrochemically, hydrothermally, or mechanically fabricated in situ with a structural phase transition have distinctive advantages, such as high charge transfer and efficient catalytic properties $^{4,22}$. However, although the photophysical properties of metallic TMD-QDs are expected to be advantageous, most studies have focused on semiconducting TMD-QDs. This is mainly due to the various synthetic routes and particular phase engineering related to the metastable metallic phase of TMDs ${ }^{6,7,21,23,24}$. Phase engineering for metallic phase TMDs has been developed via methods such as intercalation of alkali metals, electron-beam irradiation, and plasmonic hot electron injection, among others, including direct synthesis ${ }^{6,25,26}$. Exfoliation by $\mathrm{Li}$ or $\mathrm{K}$ intercalation is the most preferred method for the synthesis of metallic phase TMDs ${ }^{5,7,20,21}$, where high quality and phase stabilization are achieved simultaneously. Moreover, the phase stability is enhanced by lithiation, doping, vacancy defects, and lattice strain ${ }^{7,21,27,28}$.

In this study, we report an in-depth investigation of the photophysical properties of metallic phase $\left(1 \mathrm{~T} / 1 \mathrm{~T}^{\prime}\right) \mathrm{TMD}$ $\left(\mathrm{MX}_{2}, \mathrm{M}=\mathrm{Mo}, \mathrm{W} ; \mathrm{X}=\mathrm{S}\right.$, Se)-QDs synthesized using the $\mathrm{K}$ and $\mathrm{Na}$ intercalation method, in which the TMD- $m$ QDs were separated into two groups of different sizes ( $m$ QDsI: $\sim 3 \mathrm{~nm}, m$ QDs-II: $\sim 12 \mathrm{~nm}$ ). The metallic phase and structural properties were confirmed by high-resolution transmission electron microscopy (HR-TEM) showing an octahedral crystalline structure, novel Raman vibrational modes below $200 \mathrm{~cm}^{-1}$, and redshifted X-ray photoelectron spectroscopy (XPS) spectra. For the photophysical properties, various spectroscopic methods were employed, including excitation-dependent PL (PLE) with two resonance excitation bands and peak shifted timeresolved PL (TRPL) spectroscopy. Density functional theory (DFT) calculations revealed that the oxidation defects at the edge structure and vacancies on the basal plane play a role in controlling the electronic structure and exciton dynamics. Based on these analyses, a photoelectronic model of TMD- $m$ QDs is suggested.

\section{Results}

Synthesis and characterization

For years, we have developed the process of cointercalation and exfoliation of TMDs using alkali metal-organic compounds, such as potassium sodium tartrate tetrahydrate salt, as shown in Fig. 1 (see details in the Experimental section and Supplementary Information $)^{5}$. In this study, we adopt this method to synthesize metallic phase TMD $\left(\mathrm{MoS}_{2}, \mathrm{MoSe}_{2}, \mathrm{WS}_{2}, \mathrm{WSe}_{2}\right)$-QDs. The as-prepared TMD-salt compounds were instantly dispersed in water and sonicated for a few minutes, followed by filtration and dialysis. During the sonication process, TMD-QDs were explosively synthesized. Atomic force microscopy (AFM) and high-resolution transmission electron microscopy (HRTEM) revealed that the TMD-QDs separated into two groups have sizes of $\sim 3 \mathrm{~nm}$ (QDs-I) and $\sim 12 \mathrm{~nm}$ (QDs-II; Fig. 2a). The AFM measurement yielded a QD thickness of 1-2 nm, regardless of the lateral size, implying that most QDs are mono- or bilayers (Supplementary Fig. S1). Figure 2b shows digital photographs of QDs-I and QDs-II dispersed in water at a concentration of $0.1 \mathrm{mg} / \mathrm{ml}$, where QDs-I are significantly brighter and closer to the blue color compared to QDs-II under UV illumination. The dispersion stability is longer than 1 month, which is attributed to the partially negatively charged QDs, as examined by the zeta potential (Supplementary Fig. S2). Furthermore, HRTEM images directly demonstrate the lateral size and circular structure of the QDs (Fig. 2c and Supplementary Fig. S3). The analysis of magnified HRTEM images (inset of Fig. 2c) shows that the lattice constants of the (100) plane are $0.25,0.23,0.2$, and $0.24 \mathrm{~nm}$ for $\mathrm{MoS}_{2}, \mathrm{MoSe}_{2}, \mathrm{WS}_{2}$, and $\mathrm{WSe}_{2}$, respectively, which are slightly smaller than those reported for $2 \mathrm{H}$ semiconducting TMD layers ${ }^{11,18,24,29}$. The HRTEM image of QD-II shows point defects on the basal plane that are most likely related to chalcogen or metal atom vacancies (Fig. 2d, top $)^{1,30}$. The corresponding fast Fourier transform pattern demonstrates the octahedral crystalline structure of QDs typically observed in metallic phase TMDs (Fig. 2d, bottom). The metallic 


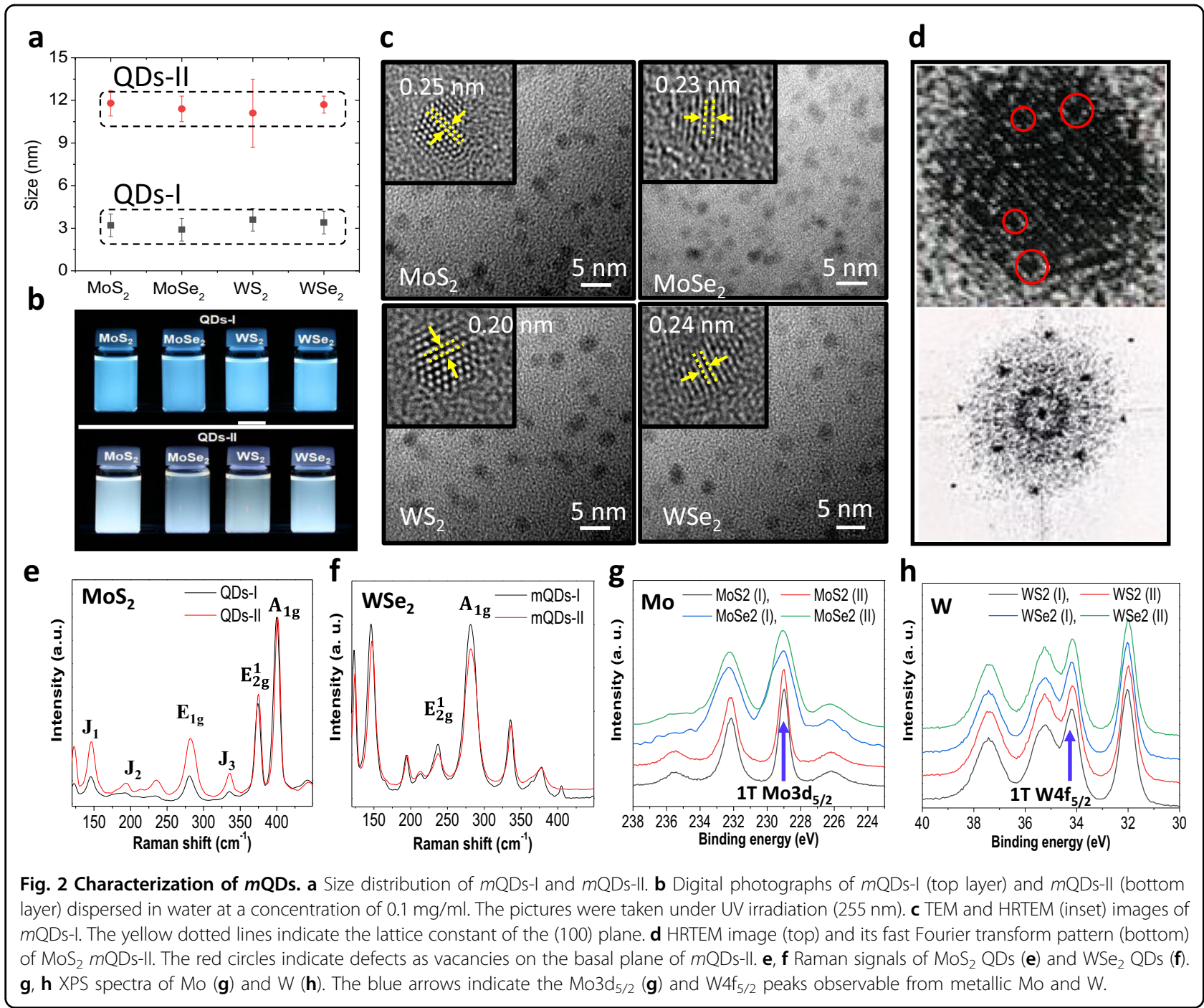

phase of the QDs is also complemented by Raman and XPS spectra (Fig. 2e, f and Supplementary Fig. S4a, b). Novel vibrational modes assigned to $J_{1}\left(146.5 \mathrm{~cm}^{-1}\right), J_{2}$ $\left(235 \mathrm{~cm}^{-1}\right)$, and $J_{3}\left(335.5 \mathrm{~cm}^{-1}\right)$ bands appear in both Raman spectra of $\mathrm{MoS}_{2}$ QDs-I and QDs-II, which are indicative of the distinct Raman signature of metallic phase $\mathrm{MoS}_{2}$ (Fig. 2e) ${ }^{7,31}$. Additionally, $\mathrm{WSe}_{2}$ QDs show distinct vibrational modes below $225 \mathrm{~cm}^{-1}$ related to the $J_{1}, J_{2}$, and $J_{3}$ transitions, while the absence of a $B^{1}{ }_{2 \mathrm{~g}}$ peak at $304 \mathrm{~cm}^{-1}$ indicates a single layer similar to $\mathrm{WSe}_{2}$ nanosheets (Fig. 2f) ${ }^{24}$. With the similar tendencies in all $\mathrm{WS}_{2}$ and $\mathrm{WSe}_{2}-\mathrm{QDs}^{32,33}$, novel vibrational modes below $200 \mathrm{~cm}^{-1}$ are noted, suggesting that the as-prepared TMD-QDs are in the metallic phase (Fig. S4a, b). In the XPS spectra of the four different TMDs-QDs (Fig. 2g, $h$ and Supplementary Fig. S4c, d), the transition metal and chalcogen peaks are shifted to lower binding energies and broadened with additional peaks compared to those of semiconducting $2 \mathrm{H}$ phase TMDs ${ }^{18,34}$. In the XPS spectra of Mo (Fig. $2 \mathrm{~g}$ and Fig. S4c), the Mo3d $\mathrm{d}_{5 / 2}$ peak is shifted $\sim 1 \mathrm{eV}$ lower compared to that measured from $2 \mathrm{H}$ semiconducting $\mathrm{MoX}_{2}$. In addition, separated W4f peaks clearly appear in the $\mathrm{W}$ spectra (Fig. $2 \mathrm{~h}$ ), and the assigned

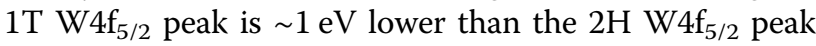
(Fig. S4d). However, the characteristic in-plane $\left(E_{2 g}^{1}\right)$ and/or out-of-plane $\left(A_{1 g}\right)$ vibrational modes in the Raman spectra and the peak shift of the metal atom to a lower binding energy in the XPS spectra indicate that the asprepared TMD-QDs could contain some portion of the polymorph structure, although the metallic phases $\left(1 \mathrm{~T} / 1 \mathrm{~T}^{\prime}\right)$ are still dominant.

\section{Electronic and optical properties}

The as-prepared TMD- $m$ QDs-I and - $m$ QDs-II were investigated by UV-visible (UV-vis) absorbance and PLE (i.e., excitation wavelength $\left(\lambda_{\mathrm{Ex}}\right)$-dependent emission wavelength $\left(\lambda_{\mathrm{Em}}\right)$ ) measurements to determine their 

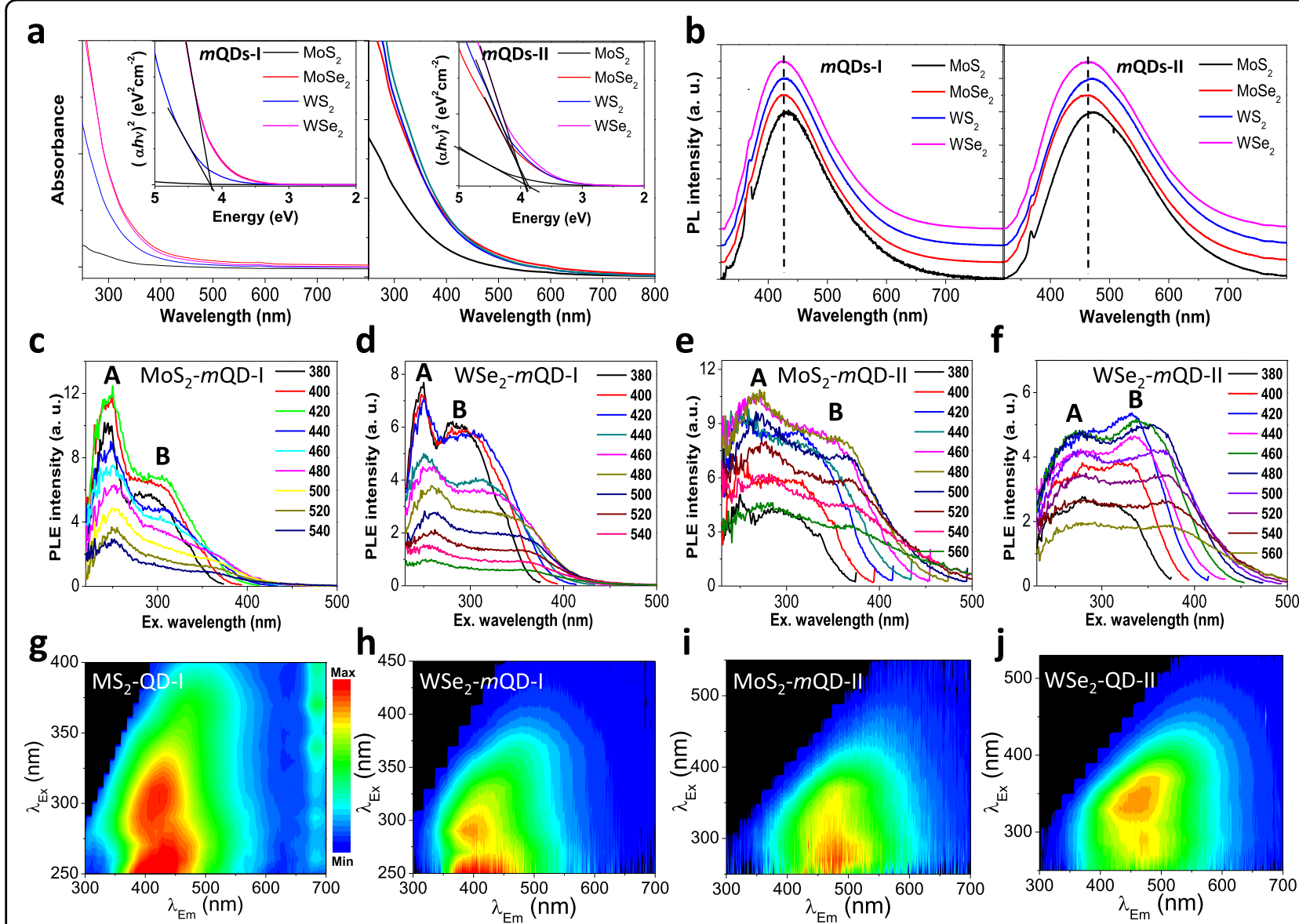

\section{e}
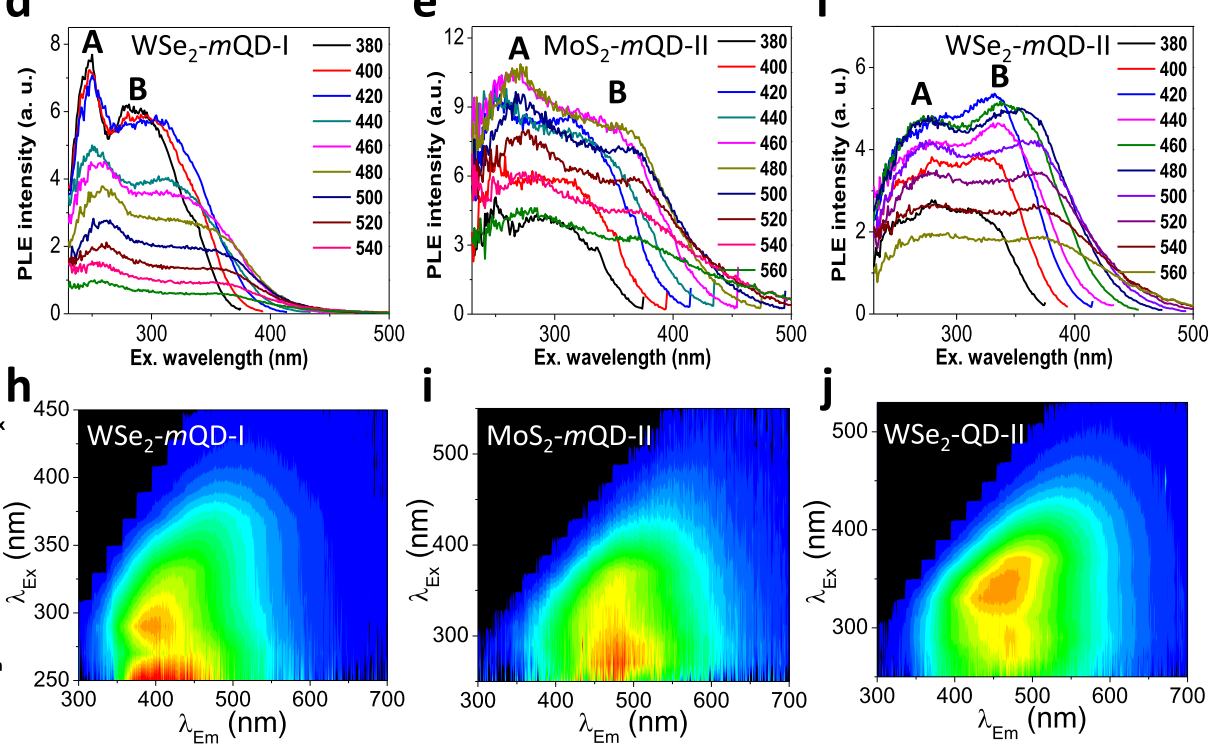

Fig. 3 Excitation wavelength-dependent emission wavelength. a, b UV absorbance (a) and PL spectra (b) of $m Q D s-I$ (left) and $m Q D s-I I$ (right). The $m Q D s-I$ were excited at $\lambda_{\text {Ex }} \sim 260 \mathrm{~nm}$, which was greater than $E_{\mathrm{og}}$, and the $m Q D$ s-II were excited at $360 \mathrm{~nm}$, which was slightly less than $E_{\mathrm{og}}$. The insets in a are Tauc plots ( $(a h v)^{2}$ vs. $\left.h v\right)$ for the optical bandgap. $\mathbf{c}-\mathbf{f}$ PLE intensity of $m$ QDs-I and $-\|$ of $\mathrm{MoS}_{2}(\mathbf{c}, \mathbf{d})$ and WSe $(\mathbf{e}, \mathbf{f})$, where the measured $\lambda_{E m}$ varies from 380 to $640 \mathrm{~nm}$. The emission wavelength $\left(\lambda_{E m}\right)$ was measured from $380 \mathrm{~nm}$ to $560 \mathrm{~nm}$. g-j. 2D contour plots of $\lambda_{\text {Ex }}$ vs. $\lambda_{E m}$ for Fig. $3 C-f$.

electronic and optical properties (Fig. 3). Figure 3a clearly shows monotonically decreasing UV-vis absorbance spectra in both $m$ QDs-I (left) and $m$ QDs-II (right), indicating the metallic properties of the QDs. The Tauc plot applied for the direct transition bandgap ( $E_{\text {og; }}$ inset of Fig. 3a) provides estimated values of $E_{\mathrm{og}}=4.14-4.20 \mathrm{eV}$, except for $\mathrm{MoSe}_{2}$, with values of $\sim 3.85 \mathrm{eV}$ in $m$ QDs-I and $3.88-3.93 \mathrm{eV}$ in $m$ QDs-II, although there is a deviation depending on the stoichiometry of the TMDs. The $E_{\mathrm{og}}$ values larger than those of bulk or single-layer TMDs are assigned to an excitonic feature of the $m$ QDs reflecting a strong quantum confinement effect ${ }^{2,11,13,16}$, whereas the difference between $m$ QDs-I and $m$ QDs-II originates from the size difference. Figure $3 \mathrm{~b}$ shows the PL spectra of $m$ QDs-I excited at $\lambda_{\mathrm{Ex}} \sim 260 \mathrm{~nm}$, which is $>E_{\mathrm{og}}$, and those of $m$ QDs-II excited at $360 \mathrm{~nm}$, which is slightly $<E_{\mathrm{og}}$. The spectral shapes are similar in each group but have different emission peaks $\left(\lambda_{\text {peak }}\right)$ and linewidths (FWHM) ( $m$ QDs-I: $\lambda_{\text {peak }}=427 \pm 3 \mathrm{~nm}(2.90 \mathrm{eV}), \mathrm{FWHM}=143 \mathrm{~nm} ; m$ QDs-II: $464 \pm 7 \mathrm{~nm}(2.67 \mathrm{eV}), \mathrm{FWHM}=184 \mathrm{~nm})$. The large Stokes shifts $(\sim 1.38 \mathrm{eV}$ and $\sim 0.78 \mathrm{eV})$ of the $m$ QDs are noteworthy and ascribed to the large valance band splitting due to the strong spin-valley coupling, midgap state caused by defects, and enormously large exciton binding energy $(>500 \mathrm{meV})$ in the $m$ QDs in addition to vibrational relaxation and trion formation ${ }^{18,35,36}$. Figure $2 \mathrm{c}-\mathrm{f}$ shows the PLE intensity of $m$ QDs-I and -II of $\mathrm{MoS}_{2}$ and $\mathrm{WSe}_{2}$, where the measured $\lambda_{\mathrm{Em}}$ varies from 380 to $640 \mathrm{~nm}$. Interestingly, in $m$ QDs-I, two persistent intensity maximum peaks clearly appear at every $\lambda_{\mathrm{Em}}$ (Fig. 3c, d). The former is located at $\lambda_{\mathrm{Ex}}=240-260 \mathrm{~nm}$ (A-band), and the latter is located at $\lambda_{\mathrm{Ex}}=290-375 \mathrm{~nm}$ (B-band). These two excitation bands are consistently observed in another $m$ QDs-I ( $\mathrm{MoSe}_{2}$ and $\mathrm{WS}_{2}$, Supplementary Fig. S5a, b). The $\lambda_{\text {Ex }}$ longer than $375 \mathrm{~nm}$ is related to the midgap states originating from defects ${ }^{18}$. The luminescence intensity peak in the A-band is independent of the stoichiometry of the TMDs for all measured $\lambda_{\mathrm{Em}}$, whereas the $\mathrm{B}$-band peak is redshifted and broadened as $\lambda_{\mathrm{Em}}$ increases. However, it is also weakly correlated with the stoichiometry 
of the TMDs. This feature is consistently observed in $m$ QDs-II, although the intensity peaks are broadened and move to the longer wavelength region (A-band: 260-285 nm, B-band: 310-390 nm; Fig. 3e, f and Supplementary Fig. S5c, d). Interestingly, in $m$ QDs-I, the PL intensity peak in the A-band is higher than that in the Bband, whereas it is similar to or smaller than that in the Bband in $m$ QDs-II. For example, in $\mathrm{WSe}_{2} m \mathrm{QDs}-\mathrm{II}$, the PL intensity in the A-band becomes like or smaller than that in the B-band (Fig. 3f). The two bands are certainly not related to the neutral free excitons or bound excitons of TMDs because of the monotonically decreasing UV absorption and single peak of the PL spectrum. This is probably due to the band splitting by strong spin-valley coupling and the quantum size effect because the $\Delta E$ between the two PLE peaks is $\sim 0.7-0.9 \mathrm{eV}$, which is like the valence band splitting in semiconducting TMD-QDs ${ }^{18}$. To date, this feature has not been reported for semiconducting TMD-QDs, which will be discussed more later. Two-dimensional contour plots for the correlation between the excitation and emission wavelengths are shown in Fig. $3 g-j$ and Supplementary Fig. S6e-h. Two regions of luminescence maximum intensity corresponding to the PLE spectra are evidently shown. The point of maximum intensity in $m$ QDs-I oscillates slightly as $\lambda_{\mathrm{Ex}}$ increases from 250 to $340 \mathrm{~nm}$, although it is gradually redshifted as $\lambda_{\mathrm{Ex}}$ increases beyond $300 \mathrm{~nm}$, which is usually observed in QDs. In contrast, the oscillation propensity of $m$ QDs-II is weakened, while the highest intensity area moves into the B-band region.

\section{Exciton dynamics}

To further investigate the exciton dynamics of the TMD- $m$ QDs, TRPL measurements were performed at two $\lambda_{\text {Ex }}$. As shown in Fig. 4a, b, the TRPL spectra of $\mathrm{WSe}_{2}$ $m$ QDs-I at $\lambda_{\mathrm{Ex}}=266 \mathrm{~nm}$ show that the PL peak gradually redshifts from $\sim 410$ to $\sim 470 \mathrm{~nm}$ as the delay time increases, whereas in $\mathrm{WSe}_{2} m \mathrm{QDs}-\mathrm{II}$, the redshift of the PL peak is trivial even after a delay time of $30 \mathrm{~ns}$. Quantitative analysis of the peak shift at $\lambda_{\mathrm{Ex}}=266 \mathrm{~nm}$ reveals that while the peak of $m$ QDs-I redshifts to above $200 \mathrm{meV}$, it is $<100 \mathrm{meV}$ in $m$ QDs-II (Fig. 4e). In comparison, the peak shift of the TRPL spectra at $\lambda_{\mathrm{Ex}}=$ $360 \mathrm{~nm}$ in both $m$ QDs-I and -II is much less than that in $\mathrm{WSe}_{2} m$ QDs-I excited at $\lambda_{\mathrm{Ex}}=266 \mathrm{~nm}$ (Fig. 4c, d). The TRPL peak shift at $\lambda_{\mathrm{Ex}}=360 \mathrm{~nm}$ is $<100 \mathrm{meV}$ in both $m$ QDs-I and -II (Fig. 4f). This feature is analogously observed in all other TMD- $m$ QDs (Supplementary Fig. S7). This suggests that some excitons formed at $\lambda_{\mathrm{Ex}}=$ $266 \mathrm{~nm}$ on $m$ QDs-I steadily transit to the lower energy states together with an emissive decay, whereas excitons formed at $\lambda_{\mathrm{Ex}}=360 \mathrm{~nm}$ on $m$ QDs-II are likely to predominantly decay at the occurring sites. This speculation is indirectly supported by the PL decay dynamics.
Although all exciton decay profiles from $m$ QDs-I and -II are best fitted by a multiexponential function, the profiles from $m$ QDs-II are closer to the monoexponential decay line (Fig. 4g). The average PL lifetime of $m$ QDs-I is $3.94 \pm$ $0.12 \mathrm{~ns}$ at $\lambda_{\mathrm{Em}}=420 \mathrm{~nm}$, which is longer than the value of $2.98 \pm 0.11 \mathrm{~ns}$ for $m$ QDs-II at $\lambda_{\mathrm{Em}}=460 \mathrm{~nm}$ (Fig. $4 \mathrm{~h}$ and Supplementary Fig. S8). This indicates that the exciton decay dynamics are different between $m$ QDs-I and -II, which can be ascribed to the different emission sites originating from different defects on the $m$ QDs.

\section{Theoretical calculation}

To prove our speculation that the electronic structure is affected by defects on TMD-mQDs, first-principles calculations within the density functional theory (DFT) framework were performed on the representative structural model of $\mathrm{MoS}_{2}$. Figure 5a shows the variation in the bandgap of $\mathrm{MoS}_{2} m$ QDs without defects with the QD size, where the bandgap increases as the size decreases due to the quantum confinement effect. However, during the synthetic process, the formation of vacancy defects on the basal plane and oxidation of edge-exposed atoms are inevitable. Figure 5b schematically illustrates the chalcogen vacancy (top) and oxygen defect (bottom) of the $\mathrm{MoS}_{2}$ structure. Structural defects are assumed based on XPS analysis (Supplementary Fig. S5). When considering such defects, the electronic bandgap of $\mathrm{MoS}_{2}$ is narrower than that of the pristine structure (Fig. 5c). Unexpectedly, with the band structure calculations (Supplementary Fig. S9), the vacancy defect on the basal plane conspicuously leads to a bandgap narrowing up to $0.2-0.4 \mathrm{eV}$ compared to that of pristine $\mathrm{MoS}_{2}$, which is a significantly larger variation than that induced by oxygen defects on the edge structure $(<0.1 \mathrm{eV})^{37}$. Furthermore, the energy difference $(0.2-0.3 \mathrm{eV})$ between them corresponds well to the difference $(\sim 0.3 \mathrm{eV})$ between the PL peaks of $m$ QDs-I and $m$ QDs-II (Fig. 3b).

\section{Discussion}

Herein, the photophysical properties of metallic phase TMD-QDs are reported by comparing $m$ QDs-I $(\sim 3 \mathrm{~nm})$ and $m$ QDs-II $(\sim 12 \mathrm{~nm})$ of two sizes. Based on the above results, a model for possible optical excitation and relaxation pathways in $m$ QDs-I and $m$ QDs-II is schematically illustrated in Fig. 6a, b. Because the $\Delta E(\sim 0.8 \mathrm{eV})$ between two PLE excitation bands (Fig. 3c-f) corresponds well to that of valence band splitting in TMD-QDs ${ }^{18}$, the system is assumed to be resonantly excited from the valence band splitting states to the conduction band ( $\mathrm{Sl}$ and higher energy states) and lower defect states. Due to the metallic phase of QDs, strongly coupled excitonplasmon polaritons might occur ${ }^{38}$, where the resonance frequency varies with the size of $m$ QDs. This, along with the defect-induced anisotropic confined excitons ${ }^{12}$, may 


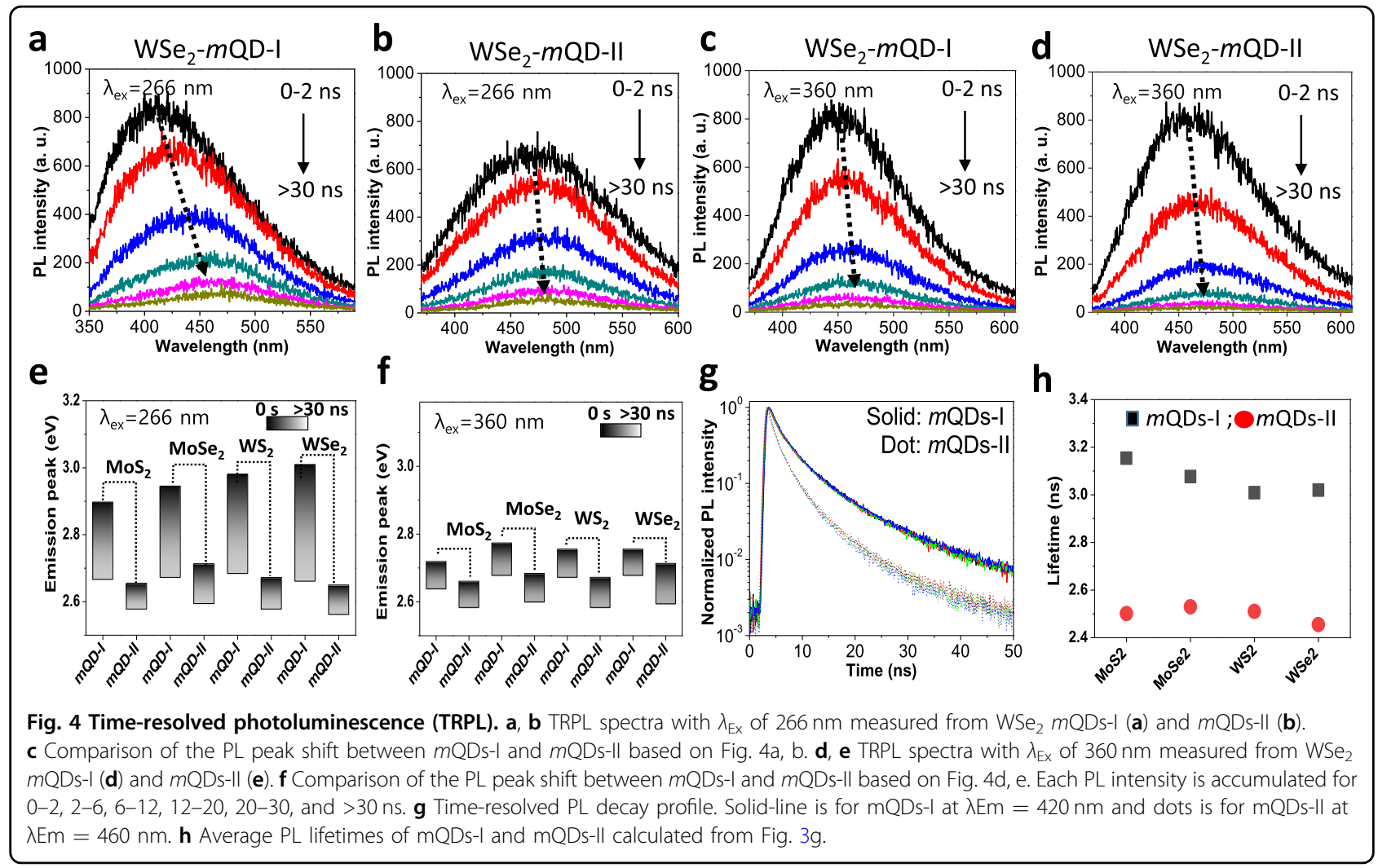

cause oscillation of the PLE intensity peak (Fig. 3g-j) and variation of the relative quantum efficiency in $m$ QDs-I and $m$ QDs-II after considering UV absorption (Supplementary Fig. S10). Additionally, as the edge oxidation of $m$ QDs-I with a size comparable to the exciton Bohr radius has a dominant effect on the electronic structure (Fig. 6a), which is in contrast to $m$ QDs-II mainly affected by vacancy defects (Fig. 6b), the radiative decay site of excitons is different from that in $m$ QDs-II, which results in the difference in the energy $(\sim 0.3 \mathrm{eV})$ and lifetime $(\sim 1 \mathrm{~ns})$ of PL. This is supported by structural analysis (Fig. 2 and Supplementary Figs. S3-5) and theoretical DFT calculations (Fig. 5). In addition, the vacancy and edge oxidation defects inducing substantial electron density localization cause a large Stokes shift (Fig. 3b) $)^{35}$.

In summary, the electronic and optical properties of metallic phase TMD-QDs ( $m$ QDs) revealed that the exciton quantum dynamics are affected by the quantum confinement effect, band splitting due to strong spinvalley coupling, and defects. Two resonance excitation bands and oscillation of the PLE intensity peak of TMD$m$ QDs are first reported, which have not yet been reported in semiconducting TMD-QDs. When the size of $m$ QDs is close to the exciton Bohr radius, edge oxidation has a dominant effect on the electronic structure; however, if the size is sufficiently larger than the exciton Bohr radius, then the vacancy on the basal plane acts as the main site determining the electronic structure and exciton dynamics. Our findings expand the general understanding of the nonlinear photophysics of two-dimensional heteroatomic structured QDs, which is essential for the potential applications of excitons-plasmons induced by light-matter interactions in optoelectronics and biooptoelectronics.

\section{Materials and methods Metallic phase TMD-QDs}

Potassium sodium tartrate was used to intercalate $\mathrm{Li}$ and $\mathrm{K}$ into the TMDs at low temperature. The metalintercalated compounds of TMDs were reacted in the autoclave vessel at $250^{\circ} \mathrm{C}$ for $12 \mathrm{~h}$ and then instantly exfoliated in water with sonication. The synthesized mQDs were separated into two sizes through filtration and dialysis5.

\section{Characterization}

The morphology of $m$ QDs was analyzed using AFM (SPA400, SII, Japan) in tapping mode under ambient conditions. UV/Vis spectra (UV-3101PC spectrometer), fluorescence spectra (Perkin-Elmer LS 55 luminescence spectrometer), XPS (Sigma Probe, AlKa), and transmission electron microscopy (TEM, Tiatan cubed G2 60-300) analyses were conducted. Raman spectra were obtained from 100 to $500 \mathrm{~cm}^{-1}$ using a Raman spectrometer (LabRAM HR 


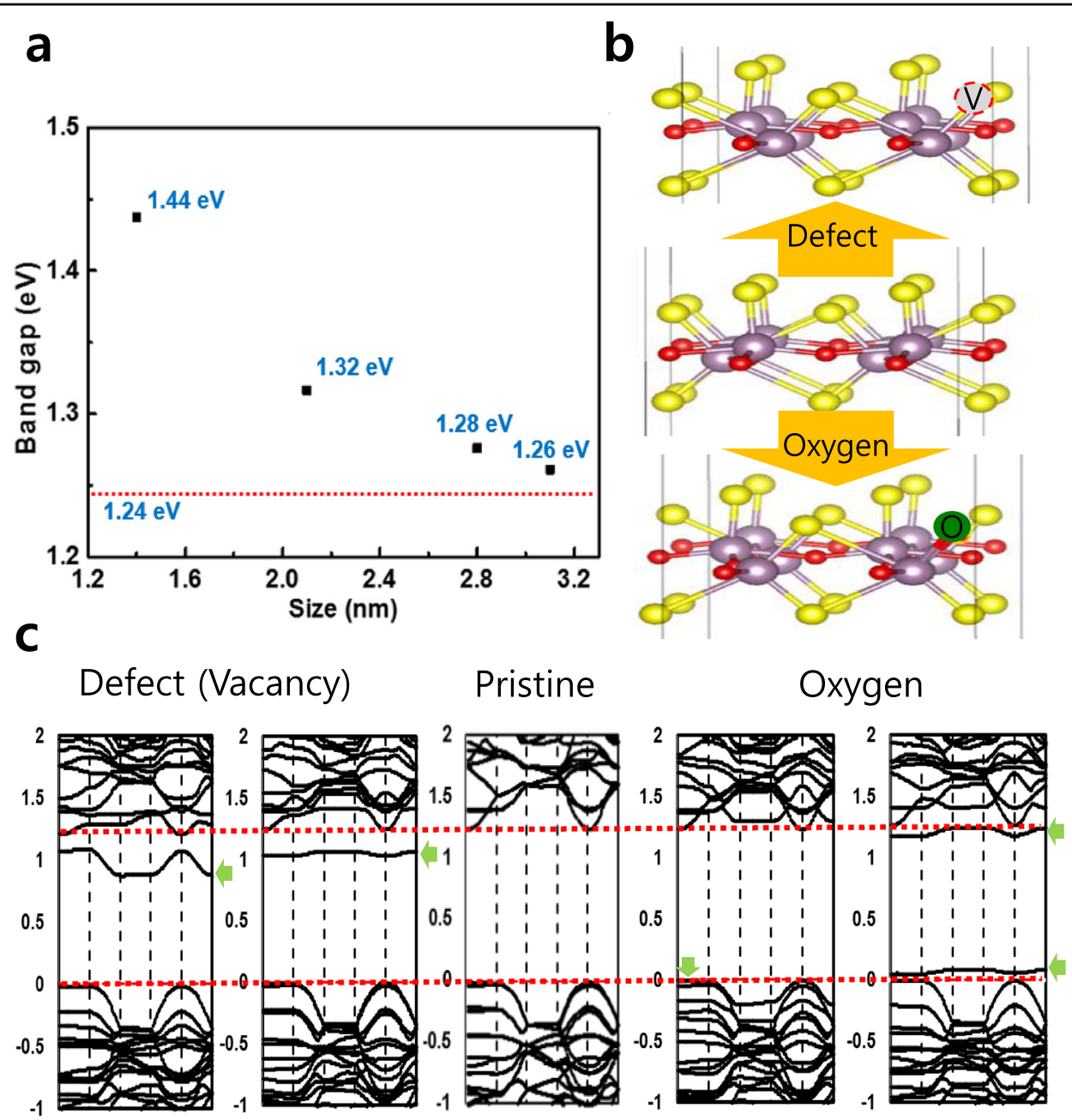

Fig. 5 Theoretical calculation. a Bandgap expansion as a function of the lateral size of mQDs. $\mathbf{b}$ Schematic illustration of the oxidation defect (bottom) and chalcogen vacancy (top). c Band structure of $\mathrm{MoS}_{2}$ with vacancy defects (left) and oxygen defects (right).
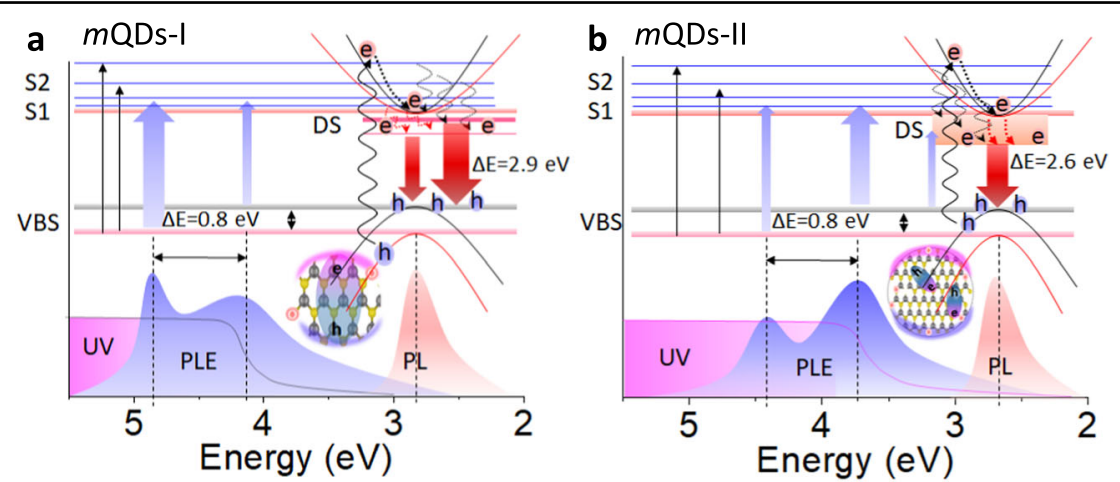

Fig. 6 Model for photophysical properties. Energy band diagram combined with UV absorption, PLE, and PL spectra of $m Q D s-I$ (a) and mQDs-II (b). 
$\mathrm{UV} / \mathrm{Vis} / \mathrm{NIR}$, excitation at $514 \mathrm{~nm}$ ). PL measurements were carried out using a 325-nm He-Cd continuous-wave laser, monochromatic light from a 300W Xenon lamp, and UV spectrometers (Maya2000, Ocean Optics, USA) as a PL detector at room temperature.

\section{Density functional calculations}

DFT calculations were performed using the generalized gradient approximation (GGA) for the exchange-correlation potential and projector augmented wave potentials, as implemented in the VASP code. The wave functions were expanded in plane waves up to an energy cutoff of $400 \mathrm{eV}$. We employed a supercell geometry with a vacuum region of more than $8 \AA$ to prohibit interactions between adjacent supercells (more details are given in the Supplementary Information).

\section{Acknowledgements}

This research was supported by the Basic Science Research Program through the National Research Foundation of Korea (NRF) funded by the Ministry of Education (2019R1A6A1A03032988). Additionally, this research was supported by the Basic Science Research Program through the National Research Foundation of Korea (NRF) founded by the Ministry of Education (NRF2020R111A307162812, NRF-2020R111A01073220). M.H.J. and Y.-H.C. were supported by the National Research Foundation (NRF-2020M3E4A1080112) of the Korean government.

\section{Author details}

'Division of Advanced Materials Engineering, Kongju National University, Chungnam 31080, South Korea. ${ }^{2}$ Department of Physics and KI for the NanoCentury, KAIST, Daejeon 34141, South Korea. ${ }^{3}$ Department of Materials Science and Engineering, Graphene Research Center of KI for the NanoCentury, KAIST, Daejeon 34141, South Korea

\section{Author contributions}

B.H.K., S.J., and S.-H.S. proposed and conducted the overall project, and M.H.J. and Y.-H.C. carried out the TRPL experiments. H.W.Y. and S.J. developed the synthetic process of MQDs. B.H.K. and S.-H.S. analyzed the data and composed the manuscript.

\section{Data availability}

The authors declare that all data supporting the findings of this study are available within the paper and its supplementary information file.

\section{Conflict of interest}

The authors declare no competing interests.

\section{Publisher's note}

Springer Nature remains neutral with regard to jurisdictional claims in published maps and institutional affiliations.

Supplementary information The online version contains supplementary material available at https://doi.org/10.1038/s41427-021-00305-z.

Received: 3 December 2020 Revised: 26 January 2021 Accepted: 19 February 2021.

Published online: 23 April 2021

\section{References}

1. Ding, $X$. et al. Defect engineered bioactive transition metals dichalcogenides quantum dots. Nat. Commun. 10, 1-13 (2019).
2. Wang, X., Sun, G., Li, N. \& Chen, P. Quantum dots derived from twodimensional materials and their applications for catalysis and energy. Chem. Soc. Rev. 45, 2239-2262 (2016).

3. Xu, Y., Wang, X., Zhang, W. L., LV, F. \& Guo, S. Recent progress in twodimensional inorganic quantum dots. Chem. Soc. Rev. 47, 586-625 (2018).

4. $\mathrm{Xu}, \mathrm{Q}$. et al. Two-dimensional quantum dots: Fundamentals, photoluminescence mechanism and their energy and environmental applications. Mater. Today Energy 10, 222-240 (2018).

5. Song, S. H. et al. Bandgap widening of phase quilted, 2D MoS2 by oxidative intercalation. Adv. Mater. 27, 3152-3158 (2015).

6. Voiry, D., Mohite, A. \& Chhowalla, M. Phase engineering of transition metal dichalcogenides. Chem. Soc. Rev. 44, 2702-2712 (2015).

7. Tan, S. J. et al. Chemical stabilization of $1 T^{\prime}$ phase transition metal dichalcogenides with giant optical Kerr nonlinearity. J. Am. Chem. Soc. 139, 2504-2511 (2017)

8. Huang, H., Fan, X., Singh, D. J. \& Zheng, W. T. Recent progress of TMD nanomaterials: phase transitions and applications. Nanoscale 12, 1247-1268 (2020).

9. Park, N.-M., Choi, C.-J., Seong, T.-Y. \& Park, S.-J. Quantum confinement in amorphous silicon quantum dots embedded in silicon nitride. Phys. Rev. Lett. 86, 1355 (2001).

10. Zhu, S. et al. The photoluminescence mechanism in carbon dots (graphene quantum dots, carbon nanodots, and polymer dots): current state and future perspective. Nano Res. 8, 355-381 (2015).

11. Gan, Z. et al. Quantum confinement effects across two-dimensional planes in MoS2 quantum dots. Appl. Phys. Lett. 106, 233113 (2015).

12. Srivastava, A. et al. Optically active quantum dots in monolayer WSe 2. Nat. Nanotechnol. 10, 491 (2015).

13. Lin, L. et al. Fabrication of luminescent monolayered tungsten dichalcogenides quantum dots with giant spin-valley coupling. ACS Nano 7, 8214-8223 (2013).

14. Xiao, D., Liu, G.B., Feng, W., Xu, X.\& Yao, W. Coupled spin and valley physics in monolayers of MoS 2 and other group-VI dichalcogenides. Phys. Rev. Lett. 108, 196802 (2012).

15. Caigas, S. P. et al. Origins of excitation-wavelength-dependent photoluminescence in WS2 quantum dots. Appl. Phys. Lett. 112, 092106 (2018).

16. Luan, C.-Y. et al. Elucidation of luminescent mechanisms of size-controllable MoSe2 quantum dots. Appl. Phys. Lett. 111, 073105 (2017).

17. Bayat, A. \& Saievar-Iranizad, E. Synthesis of blue photoluminescent WS2 quantum dots via ultrasonic cavitation. J. Lumin. 185, 236-240 (2017).

18. Zhang, K. et al. Ultrasmall and monolayered tungsten dichalcogenide quantum dots with giant spin-valley coupling and purple luminescence. ACS Omega 3, 12188-12194 (2018).

19. Jin, H. et al. Colloidal single-layer quantum dots with lateral confinement effects on 2D exciton. J. Am. Chem. Soc. 138, 13253-13259 (2016).

20. Park, S., et al. Phase engineering of transition metal dichalcogenides with unprecedentedly high phase purity, stability, and scalability via molten-metalassisted intercalation. Adv. Mater. 32, 2001889 (2020).

21. Chen, W. et al. Quantum dots of 1T phase transitional metal dichalcogenides generated via electrochemical Li intercalation. ACS Nano12 308-316 (2018).

22. Tan, C. et al. Preparation of high-percentage 1T-phase transition metal dichalcogenide nanodots for electrochemical hydrogen evolution. Adv. Mater. 30, 1705509 (2018).

23. Lin, Y.-C., Dumcenco, D. O., Huang, Y.-S. \& Suenaga, K. Atomic mechanism of the semiconducting-to-metallic phase transition in single-layered MoS 2. Nat. Nanotechnol. 9, 391-396 (2014).

24. Sokolikova, M. S., Sherrell, P. C., Palczynski, P., Bemmer, V. L. \& Mattevi, C. Direct solution-phase synthesis of 1TWSe 2 nanosheets. Nat. Commun. 10, 1-8 (2019).

25. Kang, Y. et al. Plasmonic hot electron induced structural phase transition in a MoS2 monolayer. Adv. Mater. 26, 6467-6471 (2014).

26. Friedman, A. L. et al. Evidence for chemical vapor induced $2 \mathrm{H}$ to $1 \mathrm{~T}$ phase transition in MoX $2(X=S e, S)$ transition metal dichalcogenide films. Sci. Rep. 7, 1-9 (2017).

27. Tang, Q. Tuning the phase stability of Mo-based TMD monolayers through coupled vacancy defects and lattice strain. J. Mater. Chem. C 6, 9561-9568 (2018).

28. Shang, B. et al. Lattice-mismatch-induced ultrastable 1T-phase MoS2-Pd/Au for plasmon-enhanced hydrogen evolution. Nano Lett. 19, 2758-2764 (2019). 
29. Dai, T.-J. et al. Synthesis of few-layer $2 \mathrm{H}-\mathrm{MoSe} 2$ thin films with wafer-level homogeneity for high-performance photodetector. Nanophotonics 7, 1959-1969 (2018).

30. Carozo, V. et al. Optical identification of sulfur vacancies: Bound excitons at the edges of monolayer tungsten disulfide. Sci. Adv. 3, e1602813 (2017).

31. Liu, L. et al. Phase-selective synthesis of $1 \mathrm{~T}^{\prime}$ MoS 2 monolayers and heterophase bilayers. Nat. Mater. 17, 1108-1114 (2018).

32. Pierucci, D. et al. Evidence for a narrow band gap phase in $1 \mathrm{~T}^{\prime}$ WS2 nanosheet. Appl. Phys. Lett. 115, 032102 (2019)

33. Yin, $Y$. et al. Synergistic phase and disorder engineering in 1T-MoSe2 nanosheets for enhanced hydrogen-evolution reaction. Adv. Mater. 29, 1700311 (2017).

34. Rohaizad, N., Mayorga-Martinez, C. C., Sofer, Z. K. \& Pumera, M. 1T-phase transition metal dichalcogenides (MoS2, MoSe2, WS2, and WSe2) with fast heterogeneous electron transfer: application on second-generation enzyme-based biosensor. ACS Appl. Mater. Interfaces 9, 40697-40706 (2017).

35. Liu, Y., Kim, D., Morris, O. P., Zhitomirsky, D. \& Grossman, J. C. Origins of the stokes shift in PbS quantum dots: impact of polydispersity, ligands, and defects. ACS Nano 12, 2838-2845 (2018).

36. Fan, P. et al. Trion-induced distinct transient behavior and stokes shift in WS2 monolayers. J. Phys. Chem. Lett. 10, 3763-3772 (2019).

37. Jeong, T. Y. et al. Spectroscopic studies of atomic defects and bandgap renormalization in semiconducting monolayer transition metal dichalcogenides. Nat. Commun. 10, 1-10 (2019).

38. Van Tuan, D., Scharf, B., Žutić, I. \& Dery, H. Marrying excitons and plasmons in monolayer transition-metal dichalcogenides. Phys. Rev. X 7 , 041040 (2017). 